\title{
Aortic Aneurysm with Severe Aortic Regurgitation in a Patient with Systemic Lupus Erythematosus
}

\author{
Tomoko Miyashita ${ }^{1}$, Yukio Abe ${ }^{2}$, Yasuyuki Kato ${ }^{3}$, Eiichiro Nakagawa ${ }^{2}$, Ryushi Komatsu ${ }^{2}$, \\ Koji Hattori ${ }^{3}$, Toshihiko Shibata ${ }^{3}$, Katsunobu Yoshioka ${ }^{1}$, Takahiko Naruko ${ }^{2}$ and Akira Itoh $^{2}$
}

\begin{abstract}
A 37-year-old man was admitted to our hospital for precordial chest pain. He had taken prednisolone (5 $\mathrm{mg} /$ day) for systemic lupus erythematosus (SLE) and had been symptom free for the past 12 years. Echocardiography and contrast-enhanced CT of chest showed an enlarged ascending aortic aneurysm, which is rarely seen in SLE. Severe aortic regurgitation was also present, and surgical replacement of the ascending aorta and aortic valve was successfully accomplished by the Bentall procedure. Medial cystic necrosis in the ascending aorta, which is rarely seen in SLE angiopathy, was confirmed by histology. There were no significant histopathological findings in the aortic valve.
\end{abstract}

Key words: aortic aneurysm, aortic regurgitation, systemic lupus erythematosus

(Inter Med 49: 2263-2266, 2010)

(DOI: 10.2169/internalmedicine.49.3946)

\section{Introduction}

Systemic lupus erythematosus (SLE) is an autoimmune disease that eventually results in inflammatory damage in multiple organs including skin, joints, serosa, nerves, kidneys, heart, and blood vessels (1). The cardiovascular system is often damaged, mainly as pancarditis involving the pericardium, myocardium, endocardium and coronary arteries; and there is often hypertension due to microvascular damage (2). Furthermore, valvular disease is one of the common complications, with up to 50\% of SLE patients displaying abnormalities on echocardiography (3). In contrast, macrovascular complications are infrequent in SLE, and aneurysm formation has rarely been reported $(4,5)$. Here, we report a rare case of SLE in a man complicated by ascending aortic aneurysm and severe aortic regurgitation due to aortic annular dilation.

\section{Case Report}

A 37-year-old man was admitted to our hospital because of precordial chest pain. He had a past history of SLE that included the following findings: A photosensitivity disorder was found at the age of 22 years. Two years later, a butterfly-rash and arthritis appeared accompanied by fever. Laboratory evaluation showed a WBC count $<4,000 / \mu \mathrm{L}$ and anti-Sm antibodies were positive. With these abnormal findings, he was diagnosed with SLE at the age of 24 years according to established criteria (1). Thereafter, he was treated with $40 \mathrm{mg}$ of prednisolone daily. However, one month after starting steroid therapy, he suffered from fever, headache and seizures, and was admitted to our hospital. We performed a lumbar puncture and found that the number of cells in his spinal fluid was $195 / \mathrm{mm}^{3}$ and the protein concentration was $60 \mathrm{mg} / \mathrm{dL}$. Based on these findings, he was diagnosed with lupus meningitis. A course of pulsed steroid therapy was initiated, followed by oral intake of $60 \mathrm{mg} / \mathrm{day}$ prednisolone. After discharge, the steroid dose was gradually tapered to $5 \mathrm{mg} / \mathrm{day}$ in our outpatient clinic, and he was free of SLE symptoms for the past 12 years.

At the time of his admission for precordial chest pain, physical examination revealed a blood pressure of 126/46 $\mathrm{mmHg}$, and a regular pulse of 96 beats/min. A gradeII/VI ejection systolic murmur and a II/VI diastolic murmur were noted at the left mid-sternal border and apex, respectively.

${ }^{1}$ Department of Internal Medicine, Osaka City General Hospital, Osaka, ${ }^{2}$ Department of Cardiology, Osaka City General Hospital, Osaka and ${ }^{3}$ Department of Cardiovascular Surgery, Osaka City General Hospital, Osaka Received for publication May 13, 2010; Accepted for publication July 14, 2010 Correspondence to Dr. Tomoko Miyashita, minet56@yahoo.co.jp 


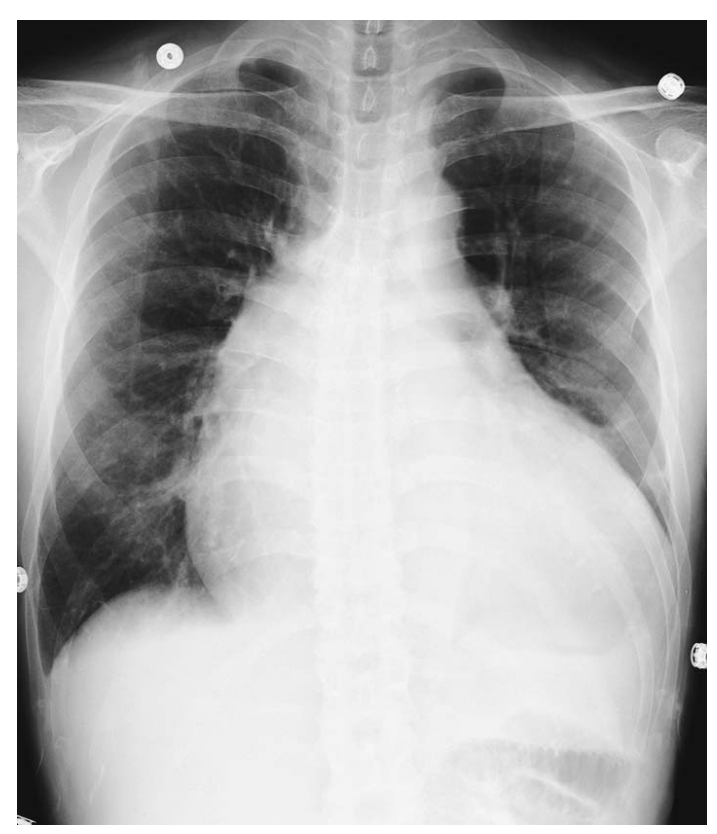

Figure 1. Chest radiograph revealed cardiomegaly but no pulmonary edema or pleural effusion.

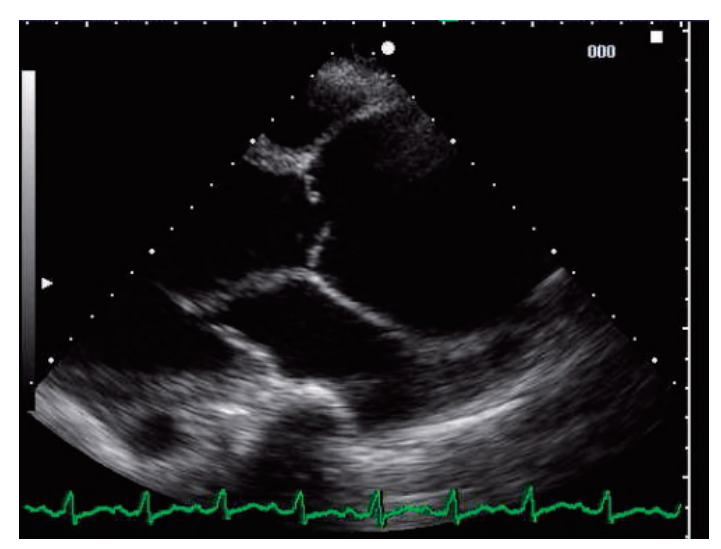

Figure 2. The two-dimensional echocardiogram showed dilation of the ascending aorta and aortic annulus.

The third and fourth heart sounds were also audible. There was no physical evidence of lupus activity in any organ system. Laboratory investigations showed the following: WBC,

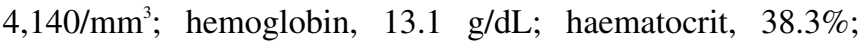
platelet count, $115 \times 10^{3} / \mathrm{mm}^{3}$; blood urea nitrogen, $10.6 \mathrm{mg} /$ $\mathrm{dL}$; creatinine, $0.99 \mathrm{mg} / \mathrm{dL}$; creatine phosphokinase $(\mathrm{CK})$, 107 IU/L; CK-MB, 9 IU/L; troponin, <0.03 ng/mL; C3, $87.3 \mathrm{mg} / \mathrm{dL} ; \mathrm{C} 4,28.2 \mathrm{mg} / \mathrm{dL}$; CRP, $1.53 \mathrm{mg} / \mathrm{dL}$; and NT prBNP, 21,106 pg/mL. A chest radiograph revealed cardiomegaly but no pulmonary edema or pleural effusion (Fig. 1). The two-dimensional echocardiogram showed dilation of the ascending aorta (diameter of $75 \mathrm{~mm}$ ) and aortic annulus (diameter of $33 \mathrm{~mm}$ ) (Fig. 2). Doppler color-flow imaging showed severe aortic regurgitation (AR) (Fig. 3). The left ventricular diastolic dimension, systolic dimension, and ejection fraction were $86 \mathrm{~mm}, 79 \mathrm{~mm}$, and $33 \%$, respectively. In addition, contrast-enhanced CT of the chest showed fusiform dilation of the ascending aorta, with a diameter of $79 \mathrm{~mm}$ (Fig. 4). According to these findings, the

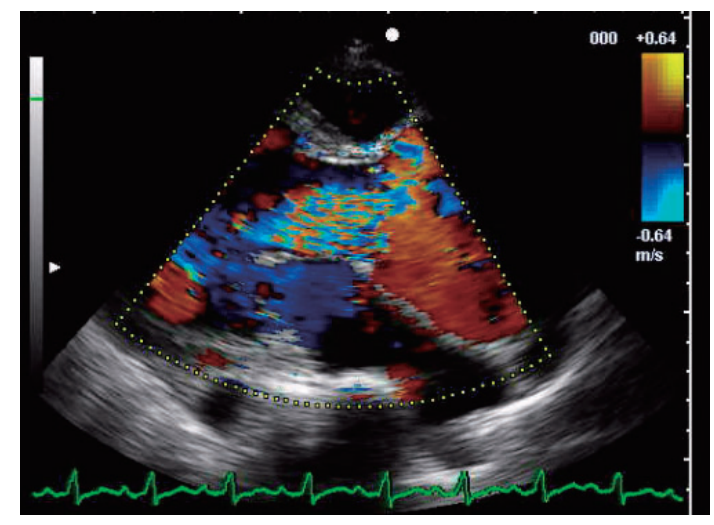

Figure 3. Doppler color-flow imaging showed a large jet of aortic regurgitation in the parasternal long-axis view.

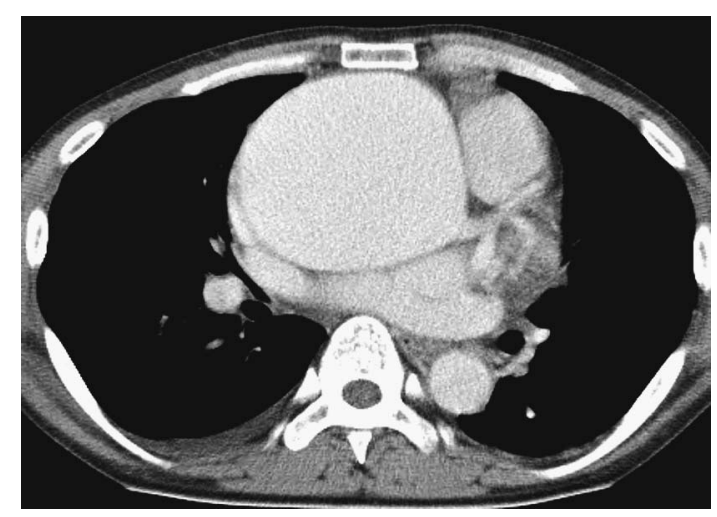

Figure 4. A contrast-enhanced CT scan of the chest showed fusiform dilation of the ascending thoracic aorta.

final diagnosis was ascending aortic aneurysm, annular dilation, AR and chronic heart failure. On the 6th day after admission, the ascending aortic aneurysm and aortic regurgitation were successfully treated with the Bentall procedure. Histopathologically, there was no evidence of active aortitis on H\&E staining (Fig. 5A). However, disruption and fragmentation of collagen fibers and the accumulation of Alcian blue-positive mucopolysaccharides were found in the ascending aortic wall, which was consistent with medial cystic necrosis (Fig. 5B and 5C). There were no significant histopathological findings in the aortic valve. His postoperative course was uneventful, and he was discharged one month after the operation with continuation of steroid therapy.

\section{Discussion}

Cardiovascular manifestations frequently complicate SLE (2). Several cardiovascular abnormalities are recognized such as hypertension and arteritis in patients with SLE. In most cases of SLE angiopathy, arteritis lesions are small vessel vasculitides and coronary arteritis. Although aortic aneurysm is a very rare complication of SLE, it can be life threatening. In 1969, Bernhard et al reported the first case of an aortic aneurysm in a patient with SLE (6). Since then, only 27 cases including dissecting aneurysm have been reported $(4,5,7-17)$. 

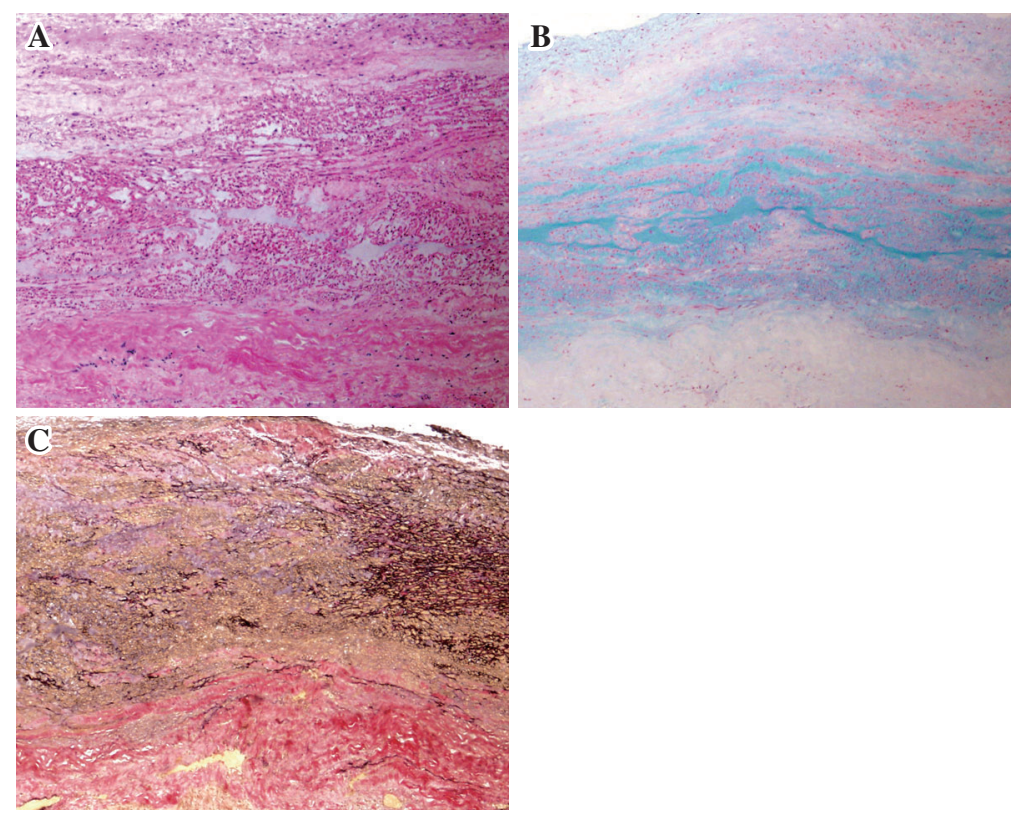

Figure 5. Histopathological findings in the ascending aortic wall. A, Hematoxylin and Eosin staining: there was no evidence of aortitis; B, Alcian blue staining: accumulation of Alcian bluepositive mucopolysaccharides; and $\mathrm{C}$, Elastica van Gieson staining: disruption and fragmentation of collagen fibers in the aortic media.

It is well known that the aneurysm formation can be caused by atherosclerosis. Aneurysm has a tendency to occur at a younger age in SLE patients than in others, although the occurrence of aneurysm is rare in SLE patients (4). Thus, other factors in addition to atherosclerosis are presumed to contribute to aneurysm formation in SLE patients. Several lines of accumulating evidence suggest that mucoid degeneration, vasculitic damage, hypertension, atherosclerosis, and steroid treatment are associated with aneurysm formation in SLE patients (10). Long-term steroid therapy leads to connective tissue weakness by suppressing the formation of granulation tissue and chondroitin sulfate, eventually leading to aneurysm formation $(10,11,18)$.

Valvular abnormalities are often detected in SLE patients. Aortic regurgitation may occur as a result of endocarditis, fenestrated aortic valve from mucoid degeneration, noninfective vegetation as a result of Libman-Sacks endocarditis and antiphospholipid antibodies (19). Non-infective vegetation was first described in 1924 by Libman and Sacks and was thought to cause valvular stenosis and regurgitation (20). Antiphospholipid antibodies are thought to cause valvular dysfunction due to platelet activation by binding to platelet phospholipids and inhibition of prostacyclin production in the endothelium. However, in the present case, noninfective vegetation on the aortic valve and antiphospholipid antibodies were not detected. The cause of severe aortic regurgitation in the present case was aortic annular dilation due to ascending aortic aneurysm.

Histopathological alterations have been reported such as medial necrosis, wide separation of elastic fibers, patchy mucoid degeneration and perivascular lymphoplasmacytic infiltrates with obliterative endarteritis of the vasa vasorum resulting in microinfarcts. These changes may be possible causes of aortic aneurysm formation in SLE patients (21). In the present case, there was no evidence of inflammatory vascular lesions. The destruction of collagen fibers and cystic medial necrosis was found, without evidence of aortitis. A decrease in the extracellular matrix leads to cystic medial necrosis. TGF- $\beta$ is crucially involved in extracellular matrix production. In SLE patients, TGF- $\beta$ production is impaired because of dysfunctional NK cells (10). Thus, an immunological disorder is presumed to contribute to aneurysm formation in SLE patients.

In summary, we describe a case of aortic aneurysm with ascending aortic aneurysm and aortic regurgitation due to annular dilation in a comparatively young SLE patient. He was treated by surgical replacement of the ascending aorta and aortic valve. It is well known that SLE patients are at risk of microvascular disease. In contrast, there are few reports of macroangiopathy such as aortic aneurysm in SLE patients. However, the actual comorbidity of macroangiopathy in patients with SLE is still unknown, and it may be larger than currently suspected. We think it may be necessary to regularly screen SLE patients for macroangiopathy.

\section{References}

1. Tan EM, Cohen AS, Fries JF, et al. The 1982 revised criteria for the classification of systemic lupus erythematosus. Arthritis Rheum 25: 1271-1277, 1982.

2. Knockaert DC. Cardiac involvement in systemic inflammatory diseases. Eur Heart J 28: 1797-1804, 2007.

3. Augoustides JG, Pochettino A, Carpenter J. Surgical management of thoracoabdominal aortic aneurysm associated with systemic lupus erythematosus. J Thorac Cardiovasc Surg 136: 215-216, 2008.

4. Ohara N, Miyata T, Kurata A, Oshiro H, Sato O, Shigematsu H. 
Ten years' experience of aortic aneurysm associated with systemic lupus erythematosus. Eur J Vasc Endovasc Surg 19: 288-293, 2000.

5. Marubayashi S, Sugi K, Ishiyama K, et al. A case of abdominal aortic aneurysm associated with systemic lupus erythematosus. Hiroshima J Med Sci 47: 85-87, 1998.

6. Bernhard GC, Lange RL, Hensley GT. Aortic disease with valvular insufficiency as the principal manifestation of systemic lupus erythematosus. Ann Intern Med 71: 81-87, 1969.

7. Kameyama K, Kuramochi S, Ueda T, et al. Takayasu's aortitis with dissection in systemic lupus erythematosus. Scand J Rheumatol 28: 187-188, 1999.

8. Foffa I, Festa PL, Ait-Ali L, Mazzone A, Bevilacqua S, Andreassi MG. Ascending aortic aneurysm in a patient with bicuspid aortic valve, positive history of systemic autoimmune diseases and common genetic factors: a case report. Cardiovasc Ultrasound 7: 34, 2009.

9. Brinster DR, Grizzard JD, Dash A. Lupus aortitis leading to aneurismal dilatation in the aortic root and ascending aorta. Heart Surg Forum 12: E105-E108, 2009.

10. Sato J, Kawakami T, Nakabayashi K, et al. Multiple aortic aneurysms complicated by a rupture in the systemic lupus erythematosus: a case report. Pathol Res Pract 204: 845-850, 2008.

11. Chang WL, Huang CM, Yang YH, Lin YT, Chiang BL. Aortic aneurysm in systemic lupus erythematosus. J Microbiol Immunol Infect 37: 310-312, 2004.

12. Nishimoto A, Haraguchi S, Kishi T, et al. A case of systemic lupus erythematosus patient who died of rupture of abdominal aortic aneurysm. Nippon Jinzo Gakkaishi 45: 701-705, 2003 (in Japanese).
13. Aoyagi S, Akashi H, Otsuka H, Sakashita H, Okazaki T, Tayama $\mathrm{K}$. Acute type A aortic dissection in a patient with systemic lupus erythematosus. Jpn Heart J 43: 567-571, 2002.

14. Kunihara T, Sasaki S, Nishibe T, et al. Successful endovascular stent-grafting for thoracic aortic aneurysms in systemic lupus erithematosus. Report of 2 cases and review of the literature. $\mathrm{J}$ Cardiovasc Surg 43: 235-240, 2002.

15. Washiyama N, Kazui T, Takinami M, et al. Surgical treatment of recurrent abdominal aortic aneurysm in a patient with systemic lupus erythematosus. J Vasc Surg 32: 209-212, 2000.

16. Murata A, Nishiya Y, Saito N, Konuma T, Yusa H, Hoshino S. De Bakey type I aortic dissection complicating systemic lupus erythematosus; report of a case. Kyobugeka 58: 902-905, 2005 (in Japanese).

17. Nosaka S, Yamauchi M, Sasaki T, Ku K, Hanada T, Tamura K. Abdominal aortic aneurysm rupture in systemic lupus erythematosus. J Cardiovasc Surg (Torino) 40: 59-61, 1999.

18. Arul Rajamurugan PS, Panchapakesa Rajendran C, Rukmangatharajan S, Kanakarani P, Rajeswari S, Ravichandran R. Aortic dissection in a case of systemic lupus erythematosus. Lupus 16: 1001-1003, 2007.

19. Cervera R, Font J, Paré C, et al. Cardiac disease in systemic lupus erythematosus: prospective study of 70 patients. Ann Rheum Dis 51: 156-159, 1992.

20. Libman E, Sacks B. A hitherto undescribed form of valvular and mural endocarditis. Arch Intern Med 33: 701-737, 1924.

21. Choi KH, Rim SJ, Lee SK, Jang BC, Cho SH. Dissecting aortic aneurysm with aortic-valve insufficiency in systemic lupus erythematosus. Nephrol Dial Transplant 14: 969-973, 1999.

(C) 2010 The Japanese Society of Internal Medicine http://www.naika.or.jp/imindex.html 